\title{
The Digital Economy: Social Interaction Technologies - an Overview
}

\author{
Teófilo Redondo
}

\author{
ZED Wordwide, Department of Research and Innovation, Madrid, Spain
}

\begin{abstract}
Social interaction technologies (SIT) is a very broad field that encompasses a large list of topics: interactive and networked computing, mobile social services and the Social Web, social software and social media, marketing and advertising, various aspects and uses of blogs and podcasting, corporate value and web-based collaboration, e-government and online democracy, virtual volunteering, different aspects and uses of folksonomies, tagging and the social semantic cloud of tags, blogbased knowledge management systems, systems of online learning, with their ePortfolios, blogs and wikis in education and journalism, legal issues and social interaction technology, dataveillance and online fraud, neogeography, social software usability, social software in libraries and nonprofit organizations, and broadband visual communication technology for enhancing social interaction. The fact is that the daily activities of many businesses are being socialized, as is the case with Yammer (https://www.yammer.com/), the social enterprise social network. The leitmotivs of social software are: create, connect, contribute, and collaborate.
\end{abstract}

Keywords - blogs, folksonomies, online learning, social interaction technologies, social media, social web, wikis

\section{INTRODUCTION AND BACKGROUND}

I $\mathrm{N}$ recent years, we have been bearing witness to an exponential growth in capabilities to electronically collect, process, store, retrieve and disseminate information and create new knowledge. This has been the case with Internet-based collaboration tools and platforms reaching end-users in unprecedented ways: online social networking, blogs, wikis, podcasts, web feeds, folksonomies, social bookmarking, photo and video sharing, discussion forums, virtual worlds, all intended to advance interaction, collaboration, and sharing online. Social Computing is the generic term used to refer to any type of computing where software serves as an intermediary for a social relation. In social computing the user takes an active role in the process, often creating content or modifying previous content, and the computing experience has extended from the individual to the social.

Social interaction technologies (SIT) and collaboration software touch many fields and they have impacted on many fields, that is to say that Web 2.0 communication converge both socially and technologically. Web science must be interdisciplinary, since it brings together experts from computer science, software engineering, management information systems, business and economics, knowledge management systems, marketing, public relations and advertising, law, journalism and media, communication, psychology, anthropology, social work, design, library and information science, and education. The new emphasis is on user-generated content, creativity, and community-based knowledge building are characteristic of Web 2.0. The term "Web 2.0" suggests a fundamental technological improvement by assigning a version number like typical IT products. Common characterizations of Web 2.0 are mostly based on seven paradigms defined by Tim O'Reilly, in his now famous blog entry What is Web 2.0 [1]: "The Web as a Platform," "Harnessing Collective Intelligence," "Data is the Next Intel Inside," "End of Software Release Cycle," "Lightweight Programming Models," "Software above the Level of a Single Device," and "Rich User Experiences."

In this "Web 2.0" world, web users have begun publishing their own content on a large scale and started using social software to store and share documents, such as photos, videos or bookmarks. The current trends indicate that a large number of US adult online consumers make daily use of social networking sites, publishing blogs/webpages, uploading visual content, commenting on blogs, posting reviews, or simply consuming user-generated content.

\section{Social media sites, 2012-2014}

$\%$ of online adults who use the following sacial media websites, by year

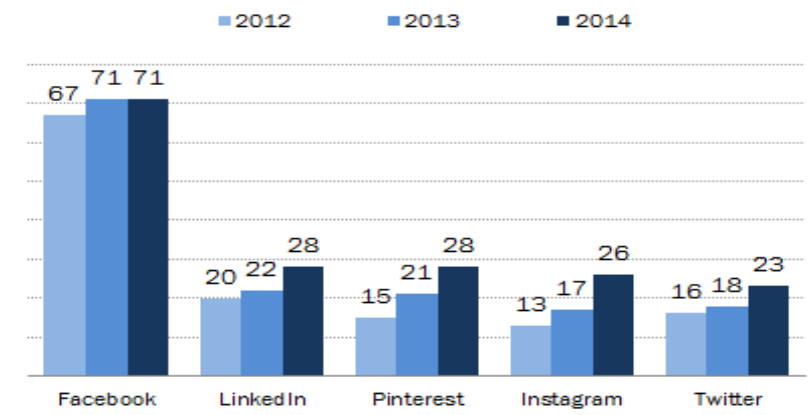

Pew Research Center's Intemet Project Surveys, 2012-2014. 2014 data collected PEW RESEARCH CENTER

Fir.1. Social Media Sites (Source: PewReseach Internet Project: http://www.pewinternet.org/2015/01/09/social-media-update-2014/)

The theoretical foundation of the social web can be traced back to J.C.R. Licklider with his seminal article "Man- 
Computer Symbiosis," [2] published in 1960, the first of three articles that attempted to redefine the human-computer interaction. Licklider outlined a vision for interactive, networked computing and, ultimately, the Internet that we experience today.

Licklider's career did not originate in computing; his studies began in "physiological psychology," the field known today as neuroscience. Licklider investigated the brain's ability to understand speech in the presence of signal distortion [3]. These early studies helped Licklider understand the workings of the human brain and prepared him to foresee the potential for improved human-computer interactions, both individually and collectively, the very basis of the online communities that conform the Social Web.

The term "Social Web" is often used in everyday language as well as in academic literature as a synonym for "virtual" and "online communities". An online community is seen as a social group that interacts through a web platform over an extended period of time. An online community can be characterized by four elements:

- group of people with shared objectives (e.g., interests, goals)

- interaction over an extended period of time

- closeness due to bonds and relationships

- shared space for interactions governed by certain rules (for example, role definitions)

The Social Web refers to an aggregation of social interaction and collaboration technologies and can be viewed as a concept and a platform that utilizes social software to support some human needs. The Social Web encompasses numerous Internet applications, such as social networking sites, blogs, podcasts, wikis, massively multiplayer online role-playing games, photo and video sharing, online stores and auction houses, simulated 3-D virtual worlds, and wiki collaborations. Various attempts to provide a definition for the Social Web have resulted in three different approaches: technical, social, and economic. The technical approach focuses on the Internet as a medium or platform for a community. The sociological point of view stresses the forming and functioning of communities, whereas the economic perspective examines potential gains and intended profits [4].

MIT's Media Lab and Intel Corporation each developed two early mobile social web applications. Social Serendipity was MIT's Bluetooth-based social service meant to harness the power of mobile technology and social information. Social Serendipity facilitated social interaction among geographically proximate users by matching user profiles and then exchanging profile information with similar matches. Intel's Jabberwocky sought to monitor and broadcast a user's movement to identify "familiar strangers" and encourage a sense of urban community. Both of these technologies relied on the mobility of the devices to evaluate locational information to facilitate social connections among users.

The Social Web is realized through social software, which is a combination of various social tools within a growing ecosystem of online data and services, all joined together (aggregated) using common protocols, and Application
Programming Interface (API) methods. Social software is at the center of the so-called API economy, a collective term referring to the economic effects enabled by companies, governments, or individuals providing direct programmable access to their systems and processes through exposing specific APIs for creating larger applications and solutions.

\section{SOCIAL SOFTWARE}

Several tools are associated with social software:

1. tools allow people to participate by creating, publishing and distributing content, such as video, pictures, music and texts through the Internet.

2. social software allows people with similar interests to find one another and connect through social networking sites, such as Facebook.

3. people can coordinate their activities and collaborate through raising petitions and funds, and planning and conducting mobile campaigns and communities programs.

4. people can create reliable, robust, and complex products such as open source software applications such as Linux (the largest example of community development).

There are three characteristics commonly attributed to social software:

- conversational interaction between individuals or groups,

- social feedback that allows a group to rate the contributions of others.

- social networks to explicitly create and manage a digital expression of people's personal relationships.

Social software serves many purposes:

- Delivery of communication between groups

- Enabling communication between many people

- Providing gathering and sharing resources

- Delivery of collaborative collecting and indexing of information

- Providing new tools for knowledge aggregation and creation of new knowledge

- Delivery to different platforms depending on the creator, recipient, and context.

In summary, above all Social Software is about group interaction. For instance, mobile social networks allow users to connect with each other, share information, and create technologically enabled mobile communities. With the introduction of the iPhone in 2007, the public dream of mobile computing was realized. Mobile communication is becoming ubiquitous in many parts of the world today with over 4 billion mobile phone users worldwide.

Although mobile phones may lead to the atomization and privatization among users by discouraging face-to-face communication, the instant accessibility to whatever social app validates the social effects of mobile phone use. As mobile technology advances, new services for mobile phones have 
been developed, which allow people to create, develop, and strengthen social interaction.

Next we will explore a little bit further the most common Social Interaction Tools.

Number of mobile phone users worldwide from 2012 to 2018 (in billions)

The statistic shows the total number of mobile phone users worldwide from 2012 to 2018. For 2017 the source projects the number of mobile phone users to reach almost 5.3 billion.

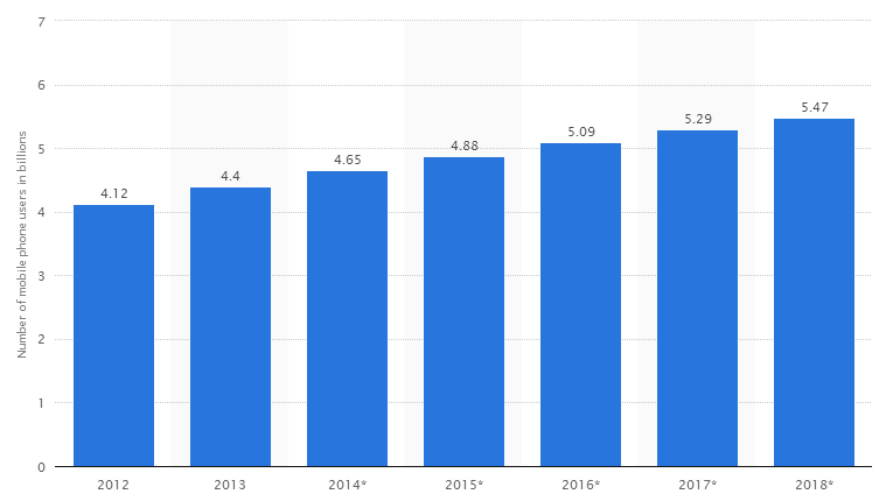

Fig. 2. Number of mobile phone (Source: Statista - The Statistics Portal: http://www.statista.com/statistics/274774/forecast-of-mobile-phone-usersworldwide/)

\section{SOCIAL INTERACTION TOOLS}

\section{A. Discussion forums}

The origin of Internet forums can be traced back to Usenet and its newsgroups, and the most recent form are the comments section at the end of articles in newspapers/magazines web sites. A forum provides an online exchange of information between people about a particular topic. It can be a place for questions and answers, or a comment-centric site and may be monitored to keep the content appropriate. Forums can be either text-only or mediarich (using images or videos to illustrate a point), and sometimes can be like a mini-portal on the topic. Forums can be entirely anonymous or require registration with username and password.

There are many types of forums: educational, professional, political, ... for instance, forums have been institutionalized as an integral part of the political communication system.

\section{B. Blogs and Wikis}

One of the main results of the Web 2.0 are new modes of online communication and self-expression. The basic form of a blog is generally accepted to be brief posts, collected on one web page, which are chronologically ordered rather than by topic or argument. Blogs have a commentary concentrated style, which may also include links, pictures, video, or any other media forms, as well as reader comments. There are two main categories: (a) filter blogs tend to be focused on external events with all kinds of links, and (b) journal blogs, a blog created and maintained by an individual where authors write about events in their own lives. Blogs can be viewed as both a technology and a communication channel.
Blogs are sometimes used by organizations as educational or knowledge management tools. Organizations are using blogs for internal information sharing and knowledge management applications. Information shared within corporate blogs may include: industry or company news, strategy brainstorming, activities within a specific department, and the sharing of customer related information. But a number of concerns have arisen, mainly a preoccupation with regards to productivity. These concerns include: a waste of work time, loss of productivity, posting of inappropriate content, reluctance of employees to share knowledge, and increasing bandwidth requirements.

A number of sites provide all the necessary tools to get started in blogging: Wordpress, Typepad or Blogger are just a few of them.

The term Wiki refers to both technology and a concept of how one can create and edit online content. Wikis are editable websites that enable users to build content and collaborate. Wikis allow users to browse through Wiki pages, edit and modify existing pages, or develop new content in a collaborative way. A Wiki is both a website and a database for keeping track of all versions of the site as modified by the users. Wikipedia [5] is probably the most well-known of wikis, but there are many, some as singular as the Intellipedia [6], a wiki for sharing restricted information among intelligence services in the United States.

Ward Cunningham conceived the first Wiki in 1994 as a series of index cards stacked upon one another, each able to be changed or altered individually without affecting the other. The format allowed for each "card" to be a new alteration to the web page and to the central database.

Wikis were first adopted by businesses as collaboration software to allow a large number of people to work on a single idea in real time, where people separated geographically could all give input into a single database using a common format. Depending on how the moderator of the Wiki sets the parameters, users can edit, add or subtract information, remain anonymous or have to login to use the system.

\section{Online social networking}

Internet has made possible for people to connect with each other beyond geographical frontiers. Social networks encompass interactions between different people, members of a community or members across different communities. Each person in this social network is represented as node or vertex and the communications represent the links or edges among these nodes. A social network comprises several focused groups or communities that can be treated as subgraphs, in order to study the structural and temporal characteristics of social networks. To separate social networks from other types of social interactions we focus here on friendship networks ("friend" in Facebook, or "follower" in Twitter, show this connectivity). In these social friendship networking sites users explicitly provide trust ratings to other members.

Social networking sites allow individuals to create online profiles providing information about themselves and their interests, create lists of users with whom they wish to share information, and to view information published within the 
network by their friends. Social networks allow us to share experiences, thoughts, opinions, and ideas.

These social interactions also led researchers to hypothesize "Small World Phenomenon" (also known as "Small World Effect"), everyone in this world can be contacted via a short chain of social acquaintances. The social psychologist Stanley Milgram in a famous experiment in 1967 set out to find the length of this short chain, and resulted in the famous concept, "six degrees of separation". This "connectedness" aspect of social interactions between people have been applied to fields as varied as genealogy studies, mathematics, economy, team sports and even corporate dynamics.

Without providing an exhaustive list of social networks, each one highlighting a special means, we can mention Facebook, Twitter, Instagram, Snapchat, Tumblr, or more specific social networks: business (LinkedIn, Xing); enterprise (Yammer); academic (ResearchGate, Gaudeamus, Academia.edu, Mendeley).

\section{Virtual worlds}

Apart from various social media (Facebook, Blogger, YouTube) Web 2.0 technologies have generated online virtual reality environments (Second Life, World of Warcraft, Sims) that have influenced today's students in many ways. There are good opportunities for immersive experiences within userconstructed environments, communities and quests. Hands-on learning (even virtual hands-on) provide an intense engagement of immersive cognitive responses.

One particular case is Massively Multiplayer Online RolePlaying Games (MMORPGs), where participants move from loose to strong associations forming social networks via structured guidelines and interaction patterns. These virtual world inhabitants create communication conduits, collaborate to attain goals and solve problems, or entertain themselves, to chart the various associations ranging from casual conversations to groups in which role specialization is critical to community success. The basis for gamification lies in using rewards for accepted behavior, then creating a socialization continuum that stimulates players to interact with one another.

The vitality of MMORPGs and MMOGs (Massively Multiplayer Online Games) assure that more MMORPGs will be on the way [8].

\section{E. Folksonomies}

Folksonomy is a portmanteau of the words folk and taxonomy. Folksonomies are a type of annotation usually referred to as social tagging, with the purpose of knowledge representation and knowledge management. Social tagging is a multidisciplinary linking knowledge representation and classification that creates an open domain network. Many recent tools and techniques focus on exploring aspects of the connection between social tagging and the underlying community, in particular the role of tagging as a means of shared informal annotation. These tags are based on user motivation and function.

Folksonomies are a relatively novel way of indexing documents and locating information based on user generated keywords. This type of grass-roots community classification (similar to other social networking approaches, such as blogs and wikis) is a good example of collective intelligence. While taxonomies are hierarchical classifications defined by formal methods that do not necessarily include user-generated tags, folksonomies structure content via user tags and the vocabulary is not preassigned. Hierarchical taxonomies attempt to organize information and give context to data through a branching structure while folksonomies allow for a multiplicity of contexts.

A folksonomy can be seen as an indexing method open for users to apply freely chosen index terms. Peter Merholz [9] entitles this method "metadata for the masses". The term "folksonomy", was introduced in 2005 by Thomas Vander Wal [10], who defines folksonomy as the outcome of individual free tagging of online content and resources in a social environment for one's own retrieval, collaboratively assigning keywords to resources or items, and sometimes have been used synonymously with the terms social classification, social indexing, or social tagging.

Large-scale social bookmarking sites (such as Del.icio.us or Reddit) have been among the earliest adopters of using folksonomies to organize information. These sites are effective tools for storing, finding, and sharing Internet-based resources, a form of social knowledge management. Much of the success of social bookmarking is attributed to its loosely structured approach to organizing data and the ease with which consumers can learn and integrate tags into a folksonomy.

\section{F. Podcasts and webcasts}

Podcasting, a portmanteau word created out of the brand name iPod and the term broadcasting is a distinctive area within social interaction technology. Content is often listened to or viewed within the world of a personal audio/video device, this more so with the ubiquitous mobile device. The upload or the download is the interaction. Podcasts are used for entertainment, education, instruction, profit and just to enjoy some time.

The first podcast took place in 2003 by automatically streaming a single audio file half way around the world. Months later Apple Computer, Inc. proved that its personal listening device, the iPod, could synchronize with a new program called iTunes and download files using the same technology. The broadcast media have begun to use podcasting as a method of time shifting programming, to allow its audience to listen to content claiming the audience consume "what they want, when they want". Educators are using podcasting for reaching out to students. Businesses are using podcasting as a marketing tool. The commercial future of podcasting appears to be in the area of advertising and broadcasting.

The user listens to or views the file, deletes it, and waits for the next episode. Because a podcast audio or video file is digital and in a format common to many devices (normally MP3), that file can go viral being reposted, edited, linked to through social sites (such as YouTube, or Facebook), or moved around through email or by some other social interaction means. 
Webcasts in the form of broadband visual communication (BVC) technologies (such as videoconferencing and video sharing) allow for the exchange of rich simultaneous or prerecorded visual and audio data over broadband networks. BVC involves both simple and complex social and technical interactions. The complexities arise as the interaction grows from communication between two individuals in the same location to communication between multiple individuals in multiple locations, working for multiple organizations located in different communities.

\section{G. Photo and video sharing}

Photo sharing can be said to be one of the first social engaging uses of the early Web 2.0 days, even before the term was coined. At a time when most cameras were not digital, users started uploading (publishing their digital photos online) and providing links for friends to share and comment, using websites like Picasa, Flickr, or Instagram.

Similarly, video sharing sites allow users to upload and share their video clips with their friends or connections (private) or with public at large (public). The best known examples are YouTube, Vimeo, and Dailymotion, as well as Netflix, Hulu, or Vine. Apart from traditional video some of these sites provide webcasting capabilities too. For instance, YouTube personal channels can be used for streaming content, which is particularly useful in the case of online learning.

\section{H. Geotagging}

Neogeography refers to geography in the Web 2.0 style, a collaborative technology from the public rather than from those in the profession, that is, a group of people (many unknown to one another) who volunteer collectively to contribute data about a topic, in this case, mapping. The practice of neogeography shares the characteristics of other social interactive technologies. Volunteer-supplied geographic tags may assume informational value beyond entertainment. Neogeography-related websites provide different ways for people to contribute photographs, locations, tags, and comments.

Neogeography might be considered a subset of cybercartography or interactive, web-based spatially referenced data, and can trace its origins to the Geospatial Web or the GeoWeb. Any sort of data that conveys place can qualify as geographical data, including for example, zip codes, area codes, images of a place, census data or place names, using a specific XML format for geographic data known as GML (Geography Markup Language).

Geosocial networking is the result of combining geotagging with social networking by including geographic services and capabilities to enable additional social dynamics, and this is the case by inserting location coordinates assigned to pictures, and then adding those pictures to maps with many applications: Flickr or Instagram for photographs, or Panoramio for Google Earth / Google Maps. Users of geosocial applications like Yelp. Facebook Places and Foursquare (Swarm) share their locations as well as ranked recommendations for locations or 'venues'.

\section{SOME SPECIAL USE CASES}

\section{A. Advertising / marketing}

Marketing is historically considered an activity that businesses performs to direct the flow of goods and services from producers to consumers. The growth of the Internet and the development of social software have changed this topdown process in the age of citizen marketing, that is, consumers voluntarily posting product information based on their knowledge and experience. Citizen marketers were envisioned by futurist Alvin Toffler [11] who coined the term "prosumers," blending the words producer and consumer, consumers who educated themselves and became involved in the design and manufacture of products. Product here refers to goods, services, brands, companies, organizations, or people, such as political candidates. The product information may take the form of opinions, reviews, videos, to be found on forums, blogs, ratings or opinion sites, social networking sites, video sharing sites, or even on mainstream marketers' websites as consumer reviews or discussion boards.

Consumers seek product information provided by citizen marketers, who are eager to share their experiences and their knowledge of a product. Citizen marketers are not on the company payroll and are not trying to sell anything. They volunteer their time as writers, animators, designers, and videographers to express their opinions about products.

\section{B. Social capital}

The social capital framework is applied to illustrate how Web 2.0 tools and techniques can support effective information and knowledge management in organizations. Managing social capital for effective knowledge sharing is a complex process, and Web 2.0 helps by creating a new culture of voluntary, contributive, and collaborative participation.

Research on social capital has been carried out in different disciplines and at different levels depending on the chosen perspective (e.g., Putnam [12]; Fukuyama [13]). Social capital includes the individual and the social aspects and is defined as the sum of actual and potential resources embedded within, available through, and derived from the network of relationships possessed by an individual or social unit. Social capital encompasses both the network and the assets that may be mobilized through that network.

Social capital is also often described in three dimensions: a structural dimension (network character), a relational dimension (trust and social identity), and a content dimension (communication to facilitate social capital). People generate economic, emotional, spiritual, and social value by engaging in social relationships. Social capital is the glue holding communities together with the power of cooperative actions. Social capital is dictated by how networks of individuals in a community create conditions where people are inclined to do things for one another.

\section{Virtual Teams}

Social interaction technologies have made it possible for teams to exist in a virtual reality. Team members can create, maintain, transmit and influence their competitiveness and 
effectiveness. The key requirements for the functioning of successful virtual teams and online culture are building trust, consolidating authentic communication flows and thinking critically.

Traditionally, a team is viewed as a group of people who bring balanced competencies to shared purposes, approaches and performance targets. There is usually synergy between the individual members of the team, which means that when the individual efforts and actions are harmonized, something different and unique is created that could not be produced by any single individual of the team. The concept of a team is expressed through seven vital elements:

1. size linked to the scope of the task

2. members' skills are balanced (basis for interdependence)

3. mutual accountability (members are synergistic and trust each other)

4. synergies of purpose

5. approach

6. performance targets

7. distance between members

\section{Online Learning}

Online behavior, distributed collaboration, and social interaction are already having a transformative effect on education, triggering changes in how teachers and students communicate and learn. Learners can engage in creative authorship by producing and manipulating digital content and making it available for consumption and critique by classmates, teachers, and a wider audience on the web.

Informal education (or learning) sits outside the traditional educational context and is voluntary, self-directed, lifelong, and motivated mainly by intrinsic interests, curiosity, exploration, and social interaction, and typically lacks the presence of an instructor. Informal learning often is self-paced and visual- or object-oriented. It provides an experiential base and motivation for further activity and learning.

The benefits and challenges of hybrid courses, which blend face-to-face instruction with online learning, leverage opportunities provided by the introduction of web-based social interaction technologies. Hybrid courses continue to evolve to meet the needs of students, instructors, and institutions of higher learning.

Today, more classrooms are equipped with various types of technology including Internet access, integrated projectors for computers, digital board, or audio and video devices. Online education management systems (Blackboard, Sakai, or Moodle), sometimes called learning management systems (LMS), are becoming more commonplace and are enabling communication, learning materials, assignments, and grading to occur online.

Early online learning environments were not engaging, and limited in supporting the interaction, coordination and cooperation between students and instructors, with low levels of confidence while learning at a distance, and low satisfaction levels resulted as a consequence. At earlier times dropout rates were relatively high. In online learning the motivation, and the sense of shared social experience are greatly constrained. New social interaction technologies can improve the social experience and social support of online learning. The members experience the online environment as a social place for learning and not in isolation. Opinion and preference of online versus classroom has turned around and now online instruction is the preferred way when a choice is offered.

\begin{tabular}{|c|c|c|c|c|c|c|}
\hline & $\begin{array}{c}\text { Total } \\
\text { Enrollment }\end{array}$ & $\begin{array}{c}\text { Annual } \\
\text { Growth Rate } \\
\text { Total } \\
\text { Enrollment }\end{array}$ & $\begin{array}{c}\text { Students } \\
\text { Taking at } \\
\text { Least One } \\
\text { Online } \\
\text { Course }\end{array}$ & $\begin{array}{l}\text { Online } \\
\text { Enrollment } \\
\text { Increase } \\
\text { over } \\
\text { Previous } \\
\text { Year }\end{array}$ & $\begin{array}{c}\text { Annual } \\
\text { Growth Rate } \\
\text { Online } \\
\text { Enrollment }\end{array}$ & $\begin{array}{l}\text { Online } \\
\text { Enrollment } \\
\text { as a Percent } \\
\text { of Total } \\
\text { Enrollment }\end{array}$ \\
\hline Fall 2002 & $16,611,710$ & NA & $1,602,970$ & NA & NA & $9.6 \%$ \\
\hline Fall 2003 & $|6,9| 1,48 \mid$ & $1.8 \%$ & 1,971,397 & 368,427 & $23.0 \%$ & $11.7 \%$ \\
\hline Fall 2004 & $17,272,043$ & $2.1 \%$ & $2,329,783$ & 358,386 & $18.2 \%$ & $13.5 \%$ \\
\hline Fall 2005 & $|7,487,48|$ & $1.2 \%$ & $3,180,050$ & 850,267 & $36.5 \%$ & $18.2 \%$ \\
\hline Fall 2006 & $17,758,872$ & $1.6 \%$ & $3,488,381$ & 308,331 & $9.7 \%$ & $19.6 \%$ \\
\hline Fall 2007 & $18,248,133$ & $2.8 \%$ & $3,938,111$ & 449,730 & $12.9 \%$ & $21.6 \%$ \\
\hline Fall 2008 & $|9,102,81|$ & $4.7 \%$ & $4,606,353$ & 668,242 & $16.9 \%$ & $24.1 \%$ \\
\hline Fall 2009 & $20,427,711$ & $6.9 \%$ & $5,579,022$ & 972,669 & $21.1 \%$ & $27.3 \%$ \\
\hline Fall 2010 & $21,016,126$ & $2.9 \%$ & $6,142,280$ & 563,258 & $10.1 \%$ & $29.2 \%$ \\
\hline Fall 2011 & $20,994,113$ & $-0.1 \%$ & $6,714,792$ & 572,512 & $9.3 \%$ & $32.0 \%$ \\
\hline Fall 2012 & $21,253,086$ & $1.2 \%$ & $7,126,549$ & 411,757 & $6.1 \%$ & $33.5 \%$ \\
\hline
\end{tabular}

Fig. 3. Online Enrollment (Source: Edudemic - Connecting education and technology: http://www.edudemic.com/2013-survey-online-learning/)

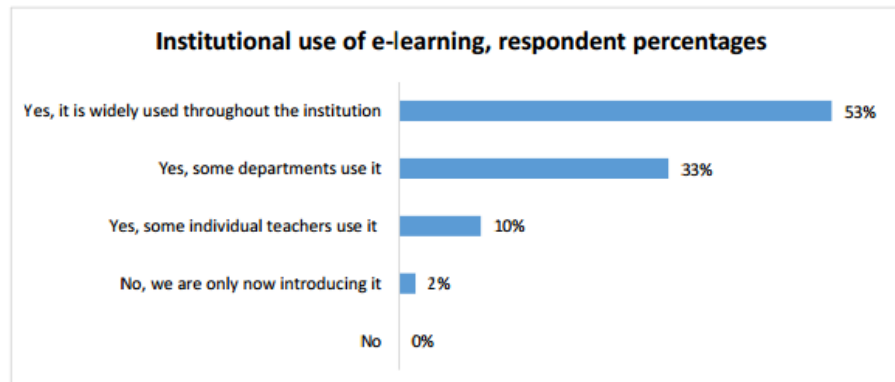

Fig. 4. (Source: European University Association :

http://www.eua.be/Libraries/Publication/e-learning_survey.sflb.ashx)

\begin{tabular}{l|ccc} 
& \multicolumn{3}{|c}{ Percent of Respondents } \\
Previous Enrollment in Online Study & All & Undergraduate & Graduate \\
\hline Yes, I took individual online courses & $45 \%$ & $41 \%$ & $45 \%$ \\
No & 43 & 51 & 37 \\
Yes, I completed another fully online program & 17 & 11 & 25 \\
& \multicolumn{3}{|c}{ Percent of Respondents } \\
Compare Online to Classroom & All & Undergraduate & Graduate \\
\hline Better & $47 \%$ & $50 \%$ & $43 \%$ \\
About the same & 43 & 41 & 48 \\
Not as good & 10 & 9 & 9
\end{tabular}

Fig. 5. (Source: Online College Students 2014:

http://www.learninghouse.com/wp-content/uploads/2014/06/2014-OnlineCollege-Students-Final.pdf)

Some examples of applying social interaction tools to education follow:

a) Wikis in education

In recent years, the field of education has discovered the educational value of the instructional use of wiki-based classroom technologies. Certain kinds of wiki-related activities correspond to certain levels of classroom interactions: social interaction, general discussion, topic-focused discussion, and collaborative/cooperative activities. Students use Wikis for 
collaborative writing exercises, or completing group assignments. [14]

\section{b) Videogames}

Squeak Etoys is a free software program and media-rich authoring system with a user-friendly visual interface. The software is designed to help six to twelve year-old children learn through interaction and collaboration. Etoys environment was created to increase the capacity for creative learning, exploration, interaction, and collaboration. Children interact, work together on projects, and engage in computer simulations and games while learning mathematics, physics, chemistry, and geometry. A similar objective is pursued by Pocket Code, strongly inspired by MIT's Scratch, the free programming language and online community that helps creating interactive stories, games, and animations.

\section{c) Webcasting}

Webcasting refers to the delivery of audio and video content over the web. The web is used as a delivery medium for informational, instructional, marketing, and entertainment purposes. Webcasting incorporates social media elements that can assist in the development of a science-oriented educational website.

\section{E. Public libraries}

Libraries have started employing social software applications (such as blogs, tagging, social networking, and wikis) to engage readers, encourage user-contributed content, and connect with user populations. User-centered philosophies are at the heart of libraries' service and have been in practice long before the emergence of Web 2.0. However, libraries have seen a radical shift as they are now faced with web-users' expectations. These expectations may not be met with less interactive computer technologies, such as library online public access catalogs (OPACs).

\section{V.CURRENT AND FUTURE TREND}

\section{A. Semantic Web}

Social tagging has become an essential element for Web 2.0 and the emerging Semantic Web applications. Web 2.0 sites express their structure, features, and relations in different ways. The model, termed the Social Semantic Cloud of Tags (SCOT), allows for the exchange of semantic tag metadata and the reuse of tags in various social software applications.

The initial purpose of tagging is to help users organize and manage their own resources, and collective tagging of common resources can be used to organize information via informal distributed groups of users. The power of semantic social tagging lies in the aggregation of information, which involves social cohesion by reinforcing social connections and providing social search mechanisms. A community built around tagging activities can be considered a social network with an insight into relations between topics and users. Semantic Web techniques and approaches help social tagging systems to eliminate tagging ambiguities.

\section{B. eGovernment and privacy in social media}

Globalization has brought a special emphasis on knowledge creation and transfer as the primary driver of economic growth and competitiveness with information technologies playing an ever-increasing role. The economic, social and political landscape in which development is taking place has changed completely.

E-government initiatives are aimed at modernizing governmental agencies in their dealings with the public and extending services into online environments. These initiatives have begun in various countries, which have allowed citizens easy access to public services and lobbying opportunities at policy level decision-making.

A number of legal issues around privacy preservation may arise from the increasing use of social interaction technologies: prospective employers searching the Internet to discover information from candidates' blogs, personal web pages, or social networking profiles; or employees being fired because of blog comments. These situations present challenges to legal systems which historically have been slow to adapt to new technologies. As a result, many of these legal issues remain unsettled.

\section{Social enterprise}

Social software is assuming a significant role in business, and has been utilized recently on a growing scale by companies in customer relationship management (CRM). A firm needs to identify the optimal level of social software deployment when planning to maximize its transactional benefits through the management of a customer knowledge base. The optimal level of social software depends on a range of factors: the initial volume of knowledge base, transaction benefits, and the estimates of the positive and negative effects of social software use.

Only recently have companies started to apply social software for managing customer knowledge, maintaining good customer relationships, and enhancing customer satisfaction, sometimes even reaching to customers in a very personalized manner. Although social software is gradually assuming a more essential role in e-business, it is still unclear at what level firms should implement it. Social software dynamically influences customer knowledge bases with direct and indirect effects of social software implementation on businesses. Customers who are dissatisfied with their shopping experiences may impact the current knowledge base affecting the transactions of future potential customers.

\section{Social Web of Things}

The relationship between Social Networks and Internet of Things (IoT) was introduced as "Social Web of Things". This idea is a redefinition of the IoT paradigm, in which things leverage social networks and specifically social standards to communicate, assigning a specific social identity to things ("smart objects") at the same level than people. Social Networks can help in that sense in "elevating" the semantics of IoT interactions to the user level and thus fostering the adoption of connected objects such as wearables, home 
automation, connected cars, to interconnect different devices with users.

"Smart social objects" consists in creating a network of "trusted" friends between humans and objects. Objects can post information to the social network, show their availability and discover new "friends", interacting with other objects or humans. In that sense the social component adds a userfriendly interaction (dialogue) paradigm for people to interact with their surrounding "Smart Social Space" environment.

Smart Social Spaces could be public, such as a local business, or private, such as a smart office or home, in which appliances and sensors communicate with one another and post their behaviors on the social wall. Users could receive (multimedia) notifications or alerts about sensors and are able to send commands remotely, for example, to their home security cam.

Some current examples of the Social Web of Things are: Toyota Friend, Nike+, Xively or Evrythng.

\section{E. Artificial Intelligence in social networks}

Facebook, Twitter, LinkedIn, and others are beginning to use artificial intelligence techniques to build their "deep learning" capacities. They are starting to process all the activity occurring over their networks, from conversations, to facial recognition, to gaming activity. Advances in cutting-edge artificial intelligence research, which program machines to perform high-level thought and abstractions, are helping social networks and their advertisers get insights from unstructured consumer data.

And one area demanding further progress is the HumanComputer symbiosis as represented by such experiments as IBM's Watson [15] and IpSoft's Amelia [16], whose stated purpose is extending a human's capabilities by applying intelligent artificial systems techniques, such as deep learning and social and interpersonal communication.

\section{F. Crowdfunding}

Crowdfunding, which can be likened to donations, is a capital collection method where common people, and not necessarily professional investors, could fund small personal or business projects by putting their own money into a kind of collective account. Originating from the evolution of Social Web technologies, crowdfunding has gained a followship because of its simplicity, and by removing more formal and traditional forms of loans, like those provided by banks, out of the picture. The most well-known crowdsourcing sites are: Kickstarter, Indiegogo, RocketHub or Razoo.

\section{G. Crowdsourcing software development}

Voluntary contribution to the creation of new software products, and amelioration of existing versions, is also a recent phenomenon originating from Social Web interaction. Crowdsourcing of software development implies the participation of large numbers of what could be termed a multidisciplinary team involving from designers, to IT architects, to code developers, to relational and documental databases administrators and developers. It is a paradigm shift from industrial mode to peer production mode with a clear impact on both time and money needed for the implementation of an IT product. By having a crowd of volunteers available, testing is much more thorough, and possible issues are detected earlier and then corrected, to the overall benefit of the community of interested parties, and consequently quality is enhanced. This collaborative software development model has now a very widespread use and all of the Free and Open Source Software (FOSS) initiatives pursue the same objectives.

One good example of crowdsourcing of software development are mashups. Loosely defined as mixing and matching content from more than one source to create a single new service displayed in a single graphical interface, the earlier uses for mashups were maps on which geolocating pictures and videos. Mashups have been made possible by the common availability of APIs to make a developer's life a lot easier.

\section{CONCLUSION}

Social Interaction Technologies (SIT) have had a transformational effect in many aspects of our lives, since they touch many fields and they have impacted on so many fields in a clear convergence both socially and technologically. Daily activities of many businesses are being socialized, incorporating the central topics of social software (create, connect, contribute, and collaborate) into a multidisciplinary ecosystem of interactive and networked computing.

We have reviewed a number of social interaction tools and some special usages where they have shown a greater effect and impact. The economic results of the so-called social media economy have yet to be produced, but only in terms of productivity increase, and employees and customers satisfaction, the value is certainly remarkable.

\section{REFERENCES}

[1] T. O'Reilly (2005). Available: http://www.oreilly.com/pub/a/web2/archive/what-is-web-20.html

[2] J.C.R. Licklider. "Man-Computer Symbiosis" (1960), IRE Transactions on Human Factors in Electronics (a copy can be accessed at http://worrydream.com/refs/Licklider\%20-\%20ManComputer\%20Symbiosis.pdf)

[3] M. Mitchell Waldrop (2001). The Dream Machine: J. C. R. Licklider and the Revolution That Made Computing Personal. New York: Viking Penguin

[4] J. Hummel, Online-Gemeinschaften als Geschäftsmodell: Eine Analyse aus sozio-ökonomischer Perspektive, 2005, Deutscher UniversitätsVerlag.

[5] Mochón, F., and M. Rojas, "IJIMAI Editor's Note - Vol. 2 Issue 5", International Journal of Interactive Multimedia and Artificial Intelligence, vol. 2, issue Special Issue on AI Techniques to Evaluate Economics and Happines, no. 5, pp. 4-5, 03/2014

[6] Calderón JCP, et al., Informe sobre la situación del Sector TIC 2010 a 2013: Mirada global y de España, Sociedad y Utopía. Revista de Ciencias Sociales 43, 84-101

[7] S. Milgram (1967) "The Small World Problem", Psychology Today, Vol. 2, 60-67

[8] MMORPG gamelist: http://www.mmorpg.com/gamelist.cfm

[9] P. Merholz (2004) Metadata for the masses. (http://www.adaptivepath.com/ideas/e000361/)

[10] T. Vander Wal (2004). Folksonomies (http://vanderwal.net/folksonomy.html)

[11] A. Toffler (1980). The Third Wave. New York: Bantam Books. 
International Journal of Artificial Intelligence and Interactive Multimedia, Vol. 3, $N^{o} 2$.

[12] R. Putnam (2000). Bowling Alone: The Collapse and Revival of American Community. New York: Simon \& Schuster

[13] F. Fukuyama (1995) Trust: The Social Virtues and the Creation of Prosperity. Cambridge University Press.

[14] W. Richardson. (2006). Blogs, wikis, podcasts, and other powerful web tools for classrooms. Thousand Oaks, CA: Corwin Press.

[15] IBM?s Watson: http://www.ibm.com/smarterplanet/us/en/ibmwatson/

[16] IpSoft's Amelia: http://www.ipsoft.com/what-we-do/amelia/

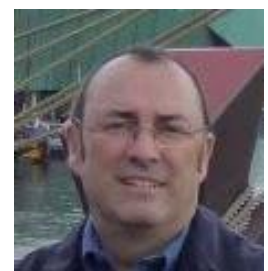

Teófilo Redondo (BArts -1985, MArts - 1986; Universidad Complutense de Madrid - UCM) is Project Portfolio Coordinator at Zed Worldwide, in the Deparment of Innovation. He was before Technology Architect \& Director of Innovation Projects at Universidad Internacional de La Rioja (UNIR). Previously he developed a career at IBM covering several areas like Cloud Computing and Big Data Architectures, Enterprise Solutions Architect (SAP, Oracle Solutions, Dassault Systemes), and as SOA Architect. He started in the Research Division of IBM with several projects on Machine Translation, during which he produced a number of articles on this subject. He was Visiting Scholar at Stanford University (1987). He currently holds an Associate Professorship at UNIR teaching about eLearning in Social Networks as well as Natural Language Processing techniques. He is affiliated with SEPLN (Sociedad Española para el Procesamiento del Lenguaje Natural) since almost the beginning. 